\title{
Ophthalmologie
}

Bitte beachten Sie: Aufgrund der Verbreitung des Coronavirus werden Veranstaltungen verschoben oder fallen kurzfristig aus. Bitte prüfen Sie vorher, ob ein Kongress stattfindet.

\author{
4. Heidelberger Helmholtztage - \\ Online- \& Präsenzveranstaltung \\ 27.-28. November 2020 \\ Wiesloch, Deutschland \\ www.klinikum.uni-heidelberg.de
}

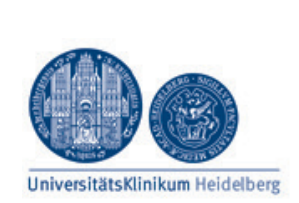

Universitătsklinikum Heidelberg
Wintertagung der Berlin-Brandenburgischen Augenärztlichen Gesellschaft BBAG

04.-05. Dezember 2020

Berlin, Deutschland

www.bbag-augen.de

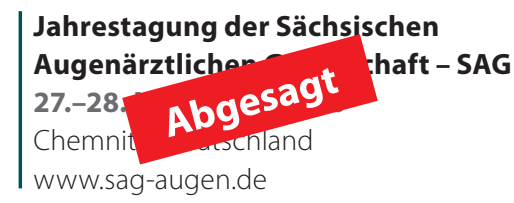

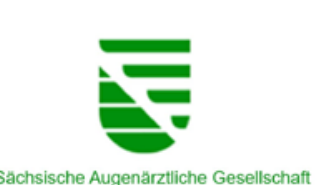

35. Kongress der Deutschsprachigen Gesellschaft für Intraokularlinsen-Implantation - DGII 2021

25.-27. Februar 2020

Dortmund, Deutschland

www.dgii.org

\section{NOVEMBER 2020}

293. Augenärzteabend in Freiburg

27. Novemba gagt

Freiburg Abgesag cutschland

www.un A

Ophthalmo Update 2020 | Berlin 10. Ophthalmologie-Update-Seminar 27.-28. November 2020

Berlin, Deutschland

ophthalmo-update.com
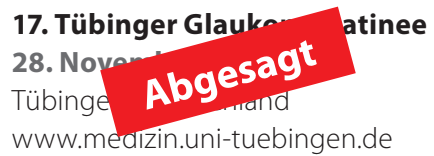

\section{DEZEMBER 2020}

Mainzer Augenärztliche Fortbildung: Kinder- und Neuroophthalmologie

02. Dezember 2020

Mainz, Deutschland

www.unimedizin-mainz.de/augenklinik

Berner Ophthalmologie-Symposium MENSCHLICHKEIT

03. Dezember 2020

Bern, Schweiz

augenklinik-bern.ch

\section{Operation Karriere}

05. Dezember 2020

Leipzig, Deutschland www.operation-karriere.de
Virtueller Kongress

14th EGS Congress 2020

European Glaucoma Society Congress

12.-13. Dezember 2020

www.egs2020.org

JANUAR 2021

Swiss Eye Week 2021

19.-22. Januar 2021

Spiez, Schweiz

swisseyeweek2021.congress-imk.ch

183. Versammlung des Vereins RheinischWestfälischer Augenärzte (RWA)

29.-30. Januar 2021

Hagen, Deutschland

\section{Operation Karriere}

30. Januar 2021

Frankfurt am Main, Deutschland

www.operation-karriere.de 